\title{
Irreducible Anterior Dislocation of the Elbow without associated Fracture
}

\author{
Gopal Prasad Gyawali, ${ }^{1}$ Bishnu Pokharel,' Rohit Kumar Pokharel' \\ 'Department of Orthopedics and Trauma Surgery, Institute of Medicine, Maharajgunj, Kathmandu, Nepal. \\ ABSTRACT
}

Anterior dislocation of the elbow joint is a rare entity and is usually associated with injuries to surrounding bony and soft tissues. Simple dislocation of the joint is managed conservatively.

An eight years old girl had traumatic anterior dislocation of the elbow joint with intact distal neurovascular status. X-rays showed no associated bony injury. Close reductions failed. Per operative findings showed no intra-articular fracture and the radial head was button holed into the anterior joint capsule. Reduction was achieved openly and maintained in a posterior slab for four weeks. Active and assisted mobilization started after removal of the slab. At ten month follow-up there was almost full range of movement of the joint.

Keywords: anterior dislocation; elbow; open reduction.

\section{INTRODUCTION}

Traumatic dislocation of the elbow in children is a rare condition. ${ }^{1-3}$ Most of the dislocations are posterior, but those can occur anteriorly, medially, laterally and rarely divergent dislocation. ${ }^{4}$ It is often occurs as a fracture-dislocation, vascular injuries and compartment syndrome. ${ }^{5-8}$ Elbow dislocations without associated fracture are termed as "simple" dislocation. Simple anterior dislocation is one of the rare injury and not well described, ${ }^{9,10}$ and reported.

Fall on the back of the elbow with the joint in flexion dislocates it anteriorly. ${ }^{11-13}$ The main pathological features are dislocation of humero-ulno-radial joint and stretching of posterior musculo-ligamentous structure and the capsule..$^{8,12}$

Most of the elbow dislocations can be reduced closely. The primary indications for open reduction are; open dislocation with arterial injuries, ${ }^{3}$ and inability to obtain a closed reduction. ${ }^{14-16}$ Here we report a rare case of irreducible "simple" anterior dislocation of the elbow joint in a girl treated surgically.

\section{CASE REPORT}

Eight years old girl presented to the hospital with complaints of swelling and deformity in right elbow for three days following fall from height. The incidence was not seen by others and the girl did not remember exact mechanism of injury. The girl had stable vitals and had no associated injury. There was no feature of generalized joint laxity. On local examination, attitude of the limb was slight flexion, elbow was swollen, deformed and tender. The relation of three bony points was distorted. Range of motion was painfully restricted with intact neurovascular status of the limb.

Routine hematological and biochemical investigations were within normal limits. Radiograph of the right elbow

Correspondence: Dr. Rohit Pokharel, Department of Orthopaedics and Trauma Surgery, Institute of Medicine, Maharaiguni, Kathmandu, Nepal. Email: pokharel.rohit@gmail.com, Phone: 9851076024. 
showed anterior dislocation of the elbow joint without fracture of surrounding bones (Figure 1).

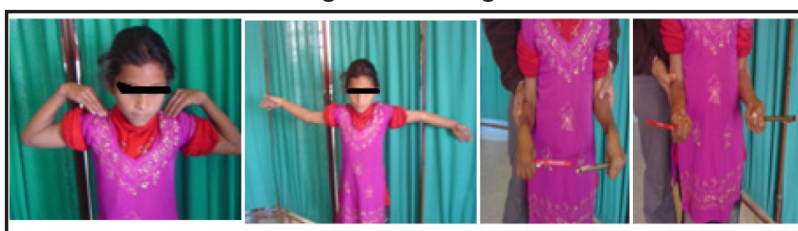

Figure 1. Radiographs before reduction. Lateral view showing complete anterior dislocation of the proximal radius and ulna. There is no associated fracture.

Closed reduction was tried twice on emergency basis under general anaesthesia, and with the help of image intensifier. The maneuver was done as recommended by Winslow $\mathrm{R}^{13}$ following these steps a) longitudinal traction along the axis of the humerus with the elbow semi-flexed to overcome the forces of the biceps and triceps; b) then the longitudinal force was applied along the axis of the forearm directed toward the elbow by flexing the elbow and pushing the forearm proximally and downward; c) reduction was tried to be accomplished by flexing the elbow and pushing the forearm proximally and downward at the same time. The maneuver was tried twice, but both the attempts were not successful.

Patient was operated on the second day of admission. Boyd and Speed approach was used to expose the elbow joint, radial head and proximal ulna. At surgery, the olecranon and the radial head were dislocated anteriorly and the radial head was button-holed through torn anterior capsule of the elbow joint hampering reduction. However, the annular ligament was slightly stretched but was intact. Part of the capsule was impinged between bony structures laterally. There was no osteo-chondral fracture in the joint. The collateral ligaments were intact.

Impinged part of the anterior capsule was excised, button-holed radial head was released and the joint was reduced under direct vision. Stability of the joint was evaluated after reduction and it was found to be stable. The reduced joint was immobilized internally with transolecranon Kirschner-wire at $90^{\circ}$ flexion and in posterior slab.

The patient was discharged on $5^{\text {th }}$ day of operation after wound inspection. On two weeks' follow-up, the wound was healed normally. The trans-olecranon Kwire and sutures were removed, and the elbow was put on posterior slab.

On second follow up (four weeks), the posterior slab was removed. Range of movement of the elbow joint was flexion $40-100^{\circ}$, supination $20^{\circ}$ and pronation $15^{\circ}$. Check radiographs showed well reduced joint (Figure 2). Patient was sent for active and assisted active ROM exercises of the elbow.

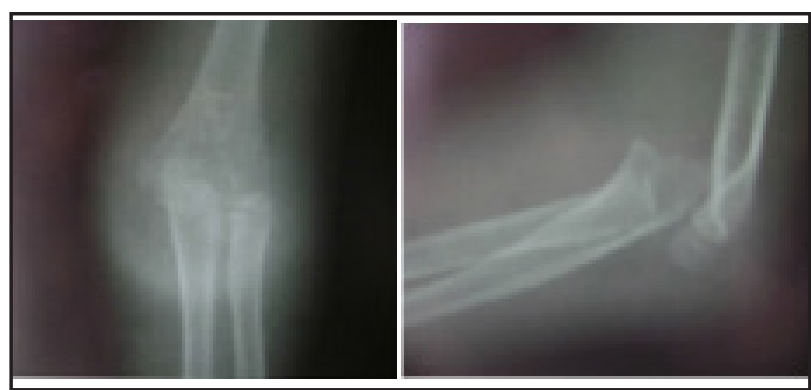

Figure 2. Radiographs, four months after open reduction, show congruous elbow joint.

In four months post-operative follow up, the ROM of the joint improved. Functionally, she could do almost all her daily activities. The radiographs showed well reduced elbow joint.

At ten months post-operative follow-up she was doing fine functionally. ROM of the elbow joint was almost full, except $20^{\circ}$ restriction in extension (Figure 4). Radiographs showed congruous joint (Figure 3).

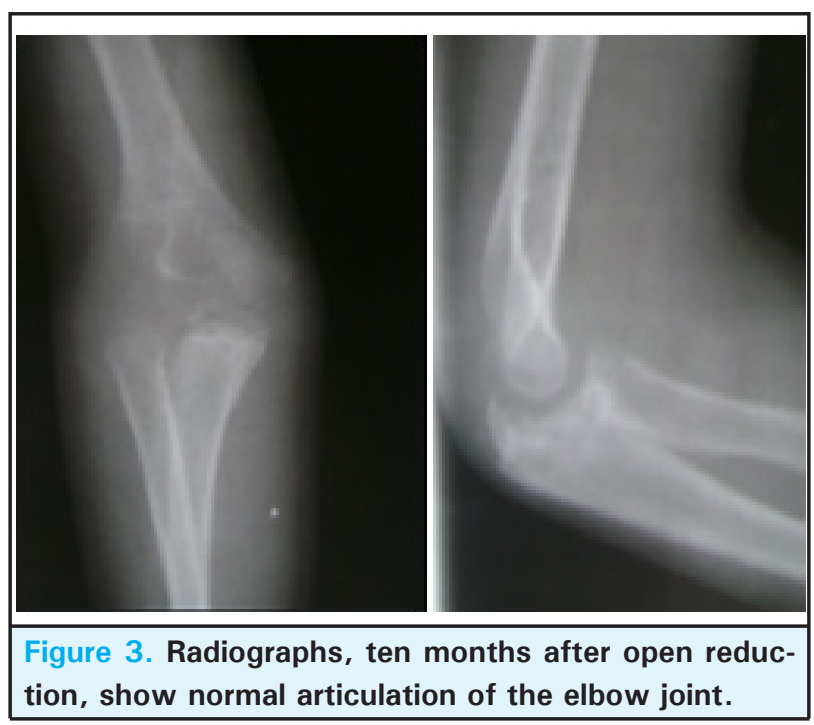




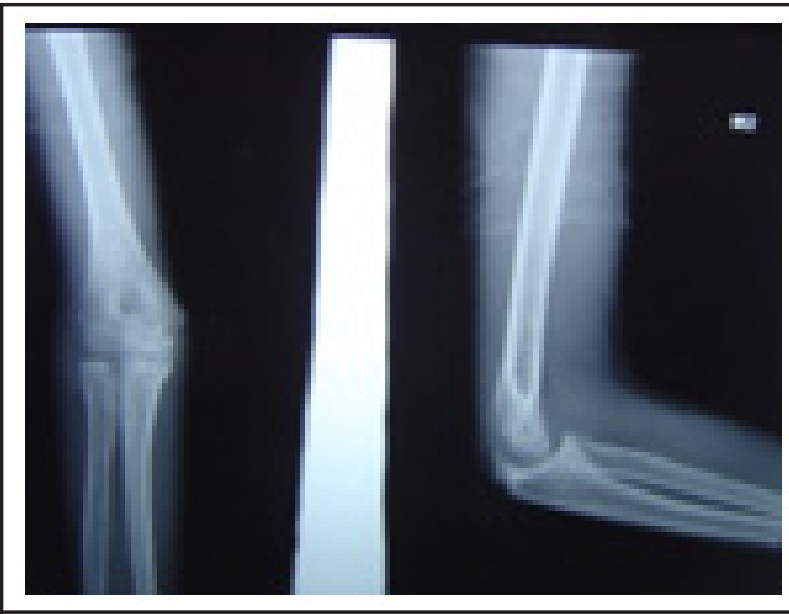

Figure 4. Photographs, at ten months latest follow up, show excellent range of movement of the elbow joint.

\section{DISCUSSION}

Anterior dislocation of the elbow is a rare injury. ${ }^{1-3,9,14-16}$ Bilateral anterior elbow dislocations have also been described in the literature, ${ }^{20}$ has even been reported after epicondylectomy ${ }^{21}$ and following childhood trauma around the elbow joint. ${ }^{22}$ The stabilizing structures of the elbow are thought to be as a ring. The posterior column is formed by the olecranon, the triceps, and the posterior part of the capsule. The trochlear notch of the proximal ulna surrounds almost 180 degrees of the trochlea, accounting for a large part of the stability of the elbow joint. Thus, the ulno-humeral articulation has been shown to be the most important stabilizer of the elbow joint. ${ }^{1,4}$ Disruption of relatively stable, posterior stabilizing structures is prerequisite for anterior dislocation of the elbow. This would explain the rarity of this injury.

In most of the reports, anterior dislocation of the elbow was associated with fractures around the elbow and in some cases there was associated neurovascular injury. ${ }^{4-7,23-25}$ In very young children even forceful pull may cause anterior dislocation of the elbow and is often misdiagnosed as "Pulled elbow". ${ }^{26}$ Most of the elbow dislocations are reduced closely, however, soft tissue interposition, ${ }^{14}$ or buttonholing of the radial head through the capsule can prevent close reduction of it. ${ }^{15,16}$ Our case is a simple or pure anterior dislocation of the elbow joint, surprisingly; close reduction was failed because of buttonholing of the radial head through the capsule of the joint. Annular ligament was intact and there was no evidence of disruption of the medial and lateral collateral ligaments. Takase $\mathrm{K}$ et al. ${ }^{14}$ from Japan and Aversano $\mathrm{F}$ et al from $\mathrm{USA}^{16}$ reported similar case of reduction failure due to buttonholing of the anterior capsule.

Regarding surgical approach and technique there is no clear recommendation in the literature. We chose the "Boyd and Speed" approach to expose the joint and for better visualization of the articular surface of the joint, radial head and proximal ulna. Reduction of the dislocated joint was possible after excising part of the impinged capsule and release of the button holed radial head. Similar surgical technique was performed to reduce the radial head from the buttonhole. ${ }^{14}$

Most authors recommend accelerated functional treatment for simple elbow dislocations, as long periods of immobilization have not been found to be of any benefit. ${ }^{24}$ In this case also we took out the transolecranon wire after two weeks and posterior slab was removed on fourth week of surgery for the purpose of early mobilization. She had good functional outcome at the time of last follow up after ten months of operation (Figure 4).

In summary, our case report is on one of the rare case, first reported from this region, of simple anterior dislocation from pediatric group which failed close reduction. Open reduction was done and the result after ten months of surgery was excellent.

\section{REFERENCES}

1. Tees FJ. Anterior dislocation at the elbow joint. Ann Surg. 1923 May;77(5):612-4.

2. Linscheid RL, Wheeler DK. Elbow dislocations. J Bone Joint Surg Am. 1965;47:1355-64.

3. Fernandez Gutierrez C, A Garciadel Couz, D Nunezbattle JA. J Troitinopicon Pazjimenez. Anterior dislocation of the elbow in children. Rev Esp Cir Osteoart. 1996;31:206-9.

4. Guitton TG, Albers RG, Ring D. Anterior olecranon fracture-dislocations of the elbow in children. A report of four cases. J Bone Joint Surg Am. 2009 Jun;91(6):1487-90.

5. Lasanianos N, Garnavos C. An Unusual Case of Elbow Dislocation: a case report. 2008;31(8):806.

6. Ring D, Jupiter JB, Sanders RW, Mast J, Simpson NS. Transolecranon fracture-dislocation of the elbow. J Orthop Trauma. 1997 Nov;11(8):545-50. 
7. Guitton TG, Albers RG, Ring D. Anterior Olecranon Fracture-Dislocations of the elbow in children: A report of four cases. J Bone Joint Surg Am. 2009 Jun;91(6):1487-90.

8. Queipo-de-Llano Temboury A, Lara JM, Fernadez-de-Rota A, Queipo-de-Llano E. Anterior elbow dislocation with potential compartment syndrome: a case report. Tech Hand Up Extrem Surg. 2007 Mar;11(1):18-23.

9. Wilkerson RD. Anterior elbow dislocation associated with olecranon fractures-review of the literature and case report. Iowa Orthop J. 1993;13:223-5.

10. A M Hidalgo Ovejero, S Garcia Mata, P Ibarra Zulategui, J Heras Izaguirre, M Martinez Grande. Anterior dislocation of the elbow without associated fracture of the olecranon: A propos of a recent case. Rev Chir Orthop Reparatrice Appar Mot. 1988;74:257-8.

11. Chbani B, Lahrach K, Amar MF, Ibnlkadi K, Elmoubaker S, Bennani A, Marzouki A, Boutayeb F. [Anterior dislocation of the elbow joint without peri-articular fracture in an adult]. Chir Main. 2012 Dec;31(6):371-3. [Article in French]

12. Panayiotis P Simonies, Nikolas C Grigoriadis, Ippokrates G Hatzokos. Anterior dislocation of the elbow: a case report. J Shoulder Elbow Surg. 2006;15(2):249-51.

13. Saouti R, Albassir A, Berger JP, Fatemi F, Willems S. Anterior elbow dislocation with recurrent instability. Acta Orthop Belg. 2003 Apr;69(2):197-200.

14. Winslow R. A case of complete anterior dislocation of both bones of the forearm at the elbow. Surg Gynecol Obstet. 1913;16:570-1.

15. Takase K, Mizuochi J. Irreducible dislocation of the radial head with undisplaced olecranon fracture in a child: a case report. J Pediatr Orthop B. 2011 Sep;20(5):345-8.

16. Takami H, Takahashi S, Ando M. Irreducible isolated dislocation of the radial head. Clin Orthop Relat Res. 1997 Dec;(345):168-70.
17. Aversano F, Kepler CK, Blanco JS, Green DW. Rare cause of block to reduction after radial head dislocation in children. J Orthop Trauma. 2011 Apr;25(4):e38-41.

18. Torchia ME, DiGiovine NM. Anterior dislocation of the elbow in an arm wrestler. J Shoulder Elbow Surg. 1998;7:539-41 .

19. Sułko J, Lejman T. Results of treatment of traumatic elbow dislocation in children. Chir Narzadow Ruchu Ortop Pol. 2000;65(1):13-8.

20. Rasool MN. Dislocations of the elbow in children. J Bone Joint Surg Br. 2004 Sep;86(7):1050-8.

21. Oury JH, Roe RD, Laning RC. A case of bilateral anterior dislocations of the elbow. J Trauma. 1972;12:170-3.

22. Cole RJ, Jemison DM, Hayes CW. Anterior elbow dislocation following medial epicondylectomy. $J$ Hand Surg [Am]. 1994;19:614-6

23. Fadili M, Wichou M, Haddoun AR, Harfaoui A, Dkhissi M, Zryouil B. Anterior luxation of the elbow. A case report. Tunis Med. 2001 Apr;79(4):251-2.

24. Jackson JA et al. Simple anterior dislocation of the elbow joint with rupture of brachial artery. Am J Surg. 1940;47:479-86.

25. Riel KA, Bernett P. [Simple elbow dislocation. Comparison of long-term results after immobilization and functional treatment]. Unfallchirurg. 1993 Oct;96:529-33.[Article in German]

26. Butler MA, Martus JE, Schoenecker JG. Pediatric variants of the transolecranon fracture dislocation: Recognition and Tension band fixation: report of 3 cases. J Hand Surg Am. 2012 May;37(5):999-1002.

27. N Venkatram, V Wurm, and S Houshian. Anterior dislocation of the ulnar-humeral joint in a so-called 'pulled elbow'. Emerg Med J. 2006 June;23(6):e37. 